Revista de Psicología Vol. 35 (2), 2017 (ISSN 0254-9247)

\title{
Caracterización del Perfeccionismo en Estudiantes con Alta Capacidad: Un Estudio de casos exploratorio
}

\author{
Andrea González Urbina ${ }^{1}$, María Paz Gómez-Arízaga², \\ María Leonor Conejeros-Solar ${ }^{3}$ \\ Pontificia Universidad Católica de Valparaíso, Chile ${ }^{1,3}$, \\ Universidad de los Andes, Chile ${ }^{2}$
}

\begin{abstract}
El perfeccionismo ha sido investigado en torno a las consecuencias que este podría significar para la salud mental de alumnos talentosos y su desarrollo socioemocional. Sin embargo, los hallazgos han sido poco concluyentes. Este estudio exploratorio apunta a descubrir las principales características del perfeccionismo adaptativo y desadaptativo en estudiantes chilenos académicamente talentosos de educación media. Para ello se detectaron casos extremos con la aplicación de la Escala Multidimensional de Perfeccionismo de Frost, para luego realizar entrevistas en profundidad semiestructuradas en una muestra de cuatro casos. Los resultados apuntan a las expectativas parentales y sociales como los principales aspectos involucrados en el desarrollo del perfeccionismo, entregando así luces de cómo apoyar la manifestación del perfeccionismo en el ámbito educativo y clínico.

Palabras clave: perfeccionismo adaptativo, perfeccionismo desadaptativo, talento académico, estudio de casos cualitativo, desarrollo socioemocional.
\end{abstract}

\section{Characterization of Perfectionism in Gifted Students: an exploratory Case Study}

Perfectionism has been investigated related to the consequences to mental health it might entail for gifted students and their socioemotional development. However, findings have been inconclusive. This exploratory study aims to discover the main characteristics of adaptive and maladaptive perfectionism in gifted Chilean secondary students. For this purpose,

1 Psicóloga clínica en la Unidad de Salud Mental y Psiquiatría del Hospital Augusto Riffart de Castro, Castro, Chile. Dirección postal: Freire 852, Castro, Chile Contacto: andreasgonzalezu@gmail.com

2 Doctora en Educación Especial por la Universidad de Arizona, Estados Unidos y profesora investigadora en la Facultad de Educación de la Universidad de los Andes. Dirección postal: Álvaro del Portillo 12.455, Las Condes, Santiago, Chile. Contacto: mpgomez@uandes.cl

3 Doctora en Educación por la Universidad de Concepción, Chile y Profesora adjunta de la Escuela de Pedagogía, Carrera de Educación Especial de la Pontificia Universidad Católica de Valparaíso. Dirección postal: Avenida El Bosque 1290, Campus Maria Teresa Brown de Ariztia, Viña del Mar, Chile. Contacto: leonor.conejeros@pucv.cl. 
extreme cases were found using Frost's Multidimensional Perfectionism Scale to subsequently conduct semi-structured interviews with a sample of four students. Results pointed to parental and societal expectations as the main aspects involved in the development of perfectionism, which sheds light on how to support the manifestation of perfectionism both in educational and clinical realms.

Key words: adaptive perfectionism, maladaptive perfectionism, gifted and talented, qualitative case study, socioemotional development.

\section{Caracterizaçáo de Perfeccionismo em Estudantes com Alta Capacidade: um Estudo de} Caso Exploratório

Apesar dos estudos sobre a cogniçấo dos alunos com Altas Habilidades ser abrangente, investigar as questôes sócioemocionais desse grupo se faz necessário. Uma das vertentes que vem sendo estudada é o perfeccionismo, pois apresenta significado para saúde mental e desenvolvimento desse grupo de pessoas. Contudo, as descobertas ainda são pouco conclusivas. Este estudo exploratório visa descobrir as principais características do perfeccionismo adaptativo e mal adaptativo em estudantes chilenos academicamente talentosos do Ensino Médio - $1^{\circ}$ a $3^{\circ}$ / Ensino Fundamental $-5^{\circ}-9^{\circ}$ através da Escala Multidimensional de Frost. Para isso, detectou-se casos extremos com a aplicação desse modelo e na sequência do trabalho foram realizadas entrevistas com profundidade em uma amostra de quatro casos. Os resultados apontam para as expectativas sociais e familiares como os principais aspectos envolvidos no desenvolvimento do perfeccionismo, tanto no âmbito educativo quanto no clínico.

Palavras-chaves: perfeccionismo adaptativo, perfeccionismo mal adaptativo, talento acadêmico, estudo de caso qualitativo, desenvolvimento socioemocional. 
La investigación en torno a la alta capacidad ha dejado en evidencia a una persona que demuestra un alto potencial en una o más áreas del conocimiento, pero que a su vez presenta necesidades especiales tanto en su desarrollo como en cuanto a la manifestación de rasgos específicos, representando un desafío para los profesionales a él vinculados (Tarazona, 2011). Uno de aquellos desafíos es la presencia de rasgos de perfeccionismo en sujetos académicamente talentosos, rasgo de personalidad que exige la utilización de estrategias específicas dentro de las prácticas tanto pedagógicas como cotidianas (Flett \& Hewitt, 2004).

La normativa chilena para la educación vigente al año 2015 (Decreto $\left.\mathrm{N}^{\circ} 170 / 2009\right)$ norma la integración, subvención económica y confección de planes educativos específicos para alumnos que presenten necesidades educativas especiales. Asimismo, existe una breve mención a la atención a estudiantes con talento en los Estándares Orientadores para Carreras de Pedagogía, que corresponde a las acciones pedagógicas a realizar por las instituciones formadoras de profesionales de la educación. Sin embargo, muchas veces tales acciones apuntan principalmente a la inclusión de alumnos con necesidades educativas especiales transitorias o permanentes, mientras que la discusión acerca de las directrices correspondientes a la educación para el talento aún presenta vacíos y se encuentra pendiente en la agenda del Ministerio de Educación y la sociedad chilena. Así, la consideración del alumnado con talentos como un grupo con necesidades educativas especiales solo tiene lugar a través de iniciativas financiadas parcialmente por el estado, principalmente a través de programas de potenciación y apoyo paralelos a la educación formal y dependientes de universidades tradicionales (Arancibia, 2009).

En relación con ello, es necesario mencionar que Latinoamérica y específicamente Chile, mantienen aún una deuda académica en torno a la investigación del perfeccionismo en la población con altas capacidades. 
Por lo anterior, el presente estudio emerge desde la necesidad de ahondar en la comprensión de los rasgos de perfeccionismo en los sujetos académicamente talentosos, con el fin de generar información coherente con el contexto chileno contemporáneo y obtener resultados aplicables dentro del sistema educativo y social de dicho país. Resulta de vital importancia que la modalidad de la investigación se centre en recoger información desde lo narrado por los jóvenes pertenecientes a este grupo en forma de significados construidos en base a la experiencia, con el fin último de poder a repensar las intervenciones psicoeducativas y contribuir a una mejor adaptación subjetiva en función de las necesidades especiales que plantea el talento académico, procurando una visión situada y contextualizada.

\section{Alta Capacidad}

La presente investigación se sostiene teóricamente en el marco propuesto por Gagné (2013), desde donde se rescata el sentido paradigmático del talento, siendo este definido como un constructo: (a) multifactorial, considerando la complejidad que hay a su base; (b) dinámico, sujeto a cambios en su manifestación de acuerdo con las influencias de determinados agentes mediadores; (c) diverso en su forma, pues existen diferentes áreas de manifestación del talento; y (d) motor de cambio, debido a su propuesta acerca de la reforma en las prácticas pedagógicas en función de atender adecuadamente las necesidades especiales del alumnado.

El talento académico resulta ser entonces aquella excepcionalidad en relación con aspectos relativos al saber académico, tanto general como en áreas específicas, cuya detección se realiza habitualmente en contextos escolares. Dentro de las descripciones generales, se identifica a los estudiantes académicamente talentosos como especialmente impetuosos, con capacidades empáticas más pronunciadas, según su medio les permita demostrarlas, y con una marcada sensibilidad hacia asuntos que pongan a prueba temas éticos y morales (Tarazona, 2011). 
En relación con ello, Colangelo, Assouline y Gross (2004) mencionan que "los jóvenes dotados tienden a ser social y emocionalmente más maduros que sus pares de la misma edad” (p.15). Sobre lo mismo, Freeman (1998) señala que los individuos con alta capacidad "son emocionalmente más fuertes que otros, con mayor productividad, mayor motivación y dinamismo, y niveles más bajos de ansiedad" (p.5).

Sin embargo, Colangelo (2002) encontró que "la depresión, la ansiedad y el aislamiento son tres de las dificultades más comunes entre los alumnos dotados" (p.2). Tal afirmación debe ser considerada bajo el contexto relacional y la acción de factores sociales, ya que dichos obstáculos suelen aparecer en condiciones específicas generadas por el contexto. Sobre este aspecto, Freeman (1998) sostiene que el origen de aquellos inconvenientes percibidos en la población académicamente talentosa podría ser atribuido principalmente al peso y efectos del entorno social sobre el sujeto y no a una problemática inherente a la población en cuestión. Segundo, la autora sugiere que podría ser la etiqueta social entregada a dichos alumnos el factor que suscitaría una reacción determinada desde el entorno, manifestándose a través de las actitudes hacia el sujeto, generando así repercusiones que provocarían un desajuste en torno a lo social. Freeman (2004) menciona además que "los problemas entre los alumnos talentosos etiquetados se correlacionaron positivamente con las dinámicas familiares; sus familias también tenían más problemas” (p.2), apoyando así la idea de que existe una estrecha vinculación entre situaciones problemáticas en el medio y en el desbalance afectivo de los alumnos etiquetados.

\section{Perfeccionismo}

Abordaje clínico del perfeccionismo. El perfeccionismo inicialmente ha sido considerado dentro de la psicología y psiquiatría como un rasgo identitario inherente a poblaciones de individuos con trastornos tales como Trastorno Obsesivo Compulsivo (TOC) y desórdenes alimenticios (Maia et al., 2009; Shafran, Cooper \& Fairburn, 2002), especialmente dentro la teoría cognitiva o racional-emotiva, vinculado 
a una tendencia patologizante y enfocada al tratamiento terapéutico en torno a las creencias irracionales, como la del apego condicionado al desempeño, temor al fracaso, la autoexigencia extrema, entre otras (Ellis \& McLaren, 2004).

Así, se define al perfeccionista como aquel sujeto "cuyos estándares se encuentran más allá de su alcance o razón, personas que se esfuerzan compulsivamente e incansablemente hacia metas imposibles y que miden su propia valía enteramente en términos de productividad y éxito" (Burns, 1980, p. 34).

Dentro de esta postura, Frost, Marten, Lahart y Rosenblate (1990) lo caracterizan como una tendencia de las personas a establecer estándares excesivamente altos, demostrar un apego más bien rígido a esos estándares y ser muy críticos en sus auto evaluaciones. Estos autores, lo conceptualizan entonces como un término multidimensional compuesto por diferentes dimensiones, las cuales pueden ser de carácter (a) interpersonal, tales como la creencia de que los padres realizan juicios y críticas acerca del desempeño y la sensación de que estos fijan altas expectativas para el sujeto; y (b) intrapersonales, que incluye preocupación y temor hacia cometer errores, necesidad excesiva de organización y orden, la fijación de altos estándares y expectativas para el propio desempeńo, y dudas sobre si las acciones son realizadas de forma correcta.

Por otro lado, Hewitt, Flett, Turnbull-Donovan y Mikail (1991) encontraron tres orientaciones principales concernientes al concepto: orientado a sí-mismo, hacia los demás, y perfeccionismo socialmente establecido. Los autores inician su investigación acerca del perfeccionismo manteniendo la idea que un alto índice de perfeccionismo tiene innegablemente consecuencias negativas para el sujeto (Flett \& Hewitt, 2004). A partir de tal teoría, se relaciona al perfeccionismo caracterizado socialmente con tendencias depresivas, en lo que mediaría un exceso de autocrítica, afectos negativos y la sobredimensión de la opinión de los demás hacia el propio desempeńo, sumado a formas desadaptativas de lidiar con el medio, como la negación de los conflictos (Christopher \& Shewmaker, 2010; Dunkley, Zuroff \& Blankstein, 2003). 
Caracterización del perfeccionismo en estudiantes con alta capacidad / González et al.

Otras conceptualizaciones del perfeccionismo. En una línea clínica paralela a lo anteriormente expuesto, se encuentran los aportes de Adler (1956), quien desde el psicoanálisis realiza una definición pionera acerca del perfeccionismo como una herramienta para alzar sentimientos de independencia y maximizar las habilidades con el fin de usarlos para el bien social.

En relación con esta conceptualización del perfeccionismo, se puede encontrar la propuesta por Hamachek (1978), quien indica que el perfeccionismo adaptativo "se encuentra asociado a características positivas, socialmente deseadas y sería adaptativo y saludable en términos de funcionamiento psicológico" (Scappatura, Arana, Elizathe \& Rutsztein, 2011, p.2) , mientras que el perfeccionismo desadaptativo conlleva una disminución del placer involucrado en la resolución de tareas, una alta preocupación por los juicios externos sobre los resultados, temor a la evaluación y "altos niveles de perfeccionismo socialmente caracterizado (es decir, la percepción de que otros demandan a uno perfección)" (Hamachek, 1978, p. 29).

Parker (1997) realiza diferentes asociaciones entre los factores propuestos por Frost y destaca que altos niveles de organización y poca preocupación por los errores, por la crítica parental, y pocas dudas sobre sus acciones se relacionaría con un perfeccionismo adaptativo, mientras que perfeccionistas con tendencias disfuncionales mantendrían alta preocupación sobre los errores, estándares personales, expectativas parentales, críticas parentales y dudas sobre sus acciones.

Posteriormente, Kreger (1999), desde la postura del humanismo, plantea que el perfeccionismo es una herramienta de potencial desarrollo, de actualización, entendiendo esto como la capacidad de actuar y obtener consecuencias de ello. Asimismo, asegura que un perfeccionismo sano se gestaría a partir de la adecuada guía en la infancia de adultos significativos, contribuyendo a la adquisición de una autoconciencia que dé luces de un perfeccionismo como herramienta de autorrealización, abriendo el debate en torno al amplio espectro de manifestaciones del perfeccionismo y sus consecuencias sobre la adaptación subjetiva. 
Otros autores también abordan las variables en interacción con el perfeccionismo y sus manifestaciones, siendo mencionados en la literatura los hallazgos en torno a la transmisión intergeneracional del perfeccionismo, donde los padres podrían tener influencia a través de modelos de crianza, de autoridad y afectivos, generando diferentes tendencias en función del nivel de adaptación y las consecuencias del perfeccionismo en el sujeto (Chan, 2008; Soenens, Elliot, Goossens, Vansteenkiste, Luyten \& Duriez, 2005; Speirs Neumeister, 2004; Stöeber, 2014).

Stöeber y Childs (2011) señala también que serían diferentes conjugaciones entre los seis factores concernientes al perfeccionismo y las tres vías de orientación del mismo los que definirían el carácter adaptativo o desadaptativo del perfeccionismo: realiza la distinción entre los "esfuerzos perfeccionistas" (perfectionistic strivings) — compuestos por las facetas relacionadas con estándares personales y la orientación hacia sí mismo del perfeccionismo- que apuntan a un perfeccionismo adaptativo, mientras que la preocupación o inquietud perfeccionista (perfectionistic concerns) —compuesta por la preocupación por los errores, dudas sobre acciones, preocupación por la evaluación de los otros acerca del propio rendimiento, y la sensación de discrepancia entre las propias expectativas y el desempeño- tendría relación con el perfeccionismo desadaptativo.

Por otro lado, Kim y Chang (2010) consideran que las percepciones que los mismos sujetos mantienen acerca de sus rasgos de personalidad perfeccionista y el discurso que estos construyan acerca de la temática, serían los determinantes al momento de juzgar si son estos o no positivos o negativos, adaptativos o no. Así, en sus estudios se menciona que el perfeccionismo adaptativo se relaciona con mayor satisfacción con la vida, la visión de los errores como instancias de superación, expectativas positivas acerca de los propios logros, motivación intrínseca, afectos positivos en torno a sí mismos y al proceso de aprendizaje, mayor adaptación al contexto estudiantil y social, y principalmente el placer desprendido del proceso de aprendizaje y de consecución de las metas (Stöeber \& Rambow, 2007). Al contrario, los perfeccionistas 
desadaptativos mantienen una relación inversa con todos los conceptos anteriores (Bellamy, 1990).

A medida que ha avanzado el conocimiento científico en el área, el perfeccionismo es considerado ya no solo como un concepto unidimensional y síntoma secundario de cuadros patológicos específicos, sino como un rasgo de personalidad complejo en su integridad, que se constituye como un constructo cuyas bases y manifestación se sitúan en el entorno y contexto cultural del sujeto. Para comprender al perfeccionismo y sus variaciones, debemos concebirlo no como una variable discreta en una dicotomía de presencia/ausencia, sino más bien como «un continuo de comportamientos y pensamientos, que tiene aspectos positivos o saludables y negativos o no saludables» (Chan, 2008, p. 3); es decir, el perfeccionismo es un rasgo fluctuante y dinámico cuya variación puede guiar a índices tanto adaptativos como desadaptativos según impliquen manifestaciones que permitan o no el bienestar mental del sujeto.

\section{Perfeccionismo y talento académico}

Es necesario destacar en este punto que existe escasa literatura latinoamericana que aborde la relación entre el talento académico y el perfeccionismo, por lo que el contenido que sienta las bases teóricas de la presente investigación se encuentra principalmente en la literatura anglosajona.

Perfeccionismo como rasgo del estudiante talentoso. Al profundizar en las investigaciones, observaciones específicas en poblaciones de alumnos académicamente talentosos los relacionan con diversos rasgos de personalidad perfeccionista, mostrando principalmente altas exigencias autoimpuestas (Kornblum \& Ainley, 2005). Un estudio pionero en la relación entre el talento académico y el perfeccionismo (Parker, 1997) deja en evidencia la variabilidad de las manifestaciones del perfeccionismo en la población de alumnos académicamente talentosos, distinguiendo tres posiciones con respecto al perfeccionismo: el perfeccionismo sano, perfeccionismo con rasgos disfuncionales y la ausencia de rasgos perfeccionistas. 
Con tal enfoque se contribuye a la desmitificación de la imagen popular del alumno académicamente talentoso, concebida como un sujeto que en toda oportunidad se muestra altamente funcional y con gran ajuste social. Dejando en claro que al momento de considerar los niveles de adaptación al entorno social y la tendencia a la excelencia, existen altos niveles de heterogeneidad, tanto entre los individuos que componen la población, como entre diferentes aspectos del perfil psicológico de cada alumno.

Perfeccionismo y talento: la influencia del contexto. También es necesario considerar los hallazgos que representan a un sujeto que se encuentra contenido dentro de los significados contextuales y culturales, existiendo la tendencia entre diferentes autores de centrarse en este aspecto del perfeccionismo, proponiendo que tanto el talento académico como aquel concepto son construidos socialmente por los sujetos académicamente talentosos y perfeccionistas, bajo la influencia del entramado cultural y de diferentes circunstancias ambientales.

Dentro de este análisis, existe evidencia que relaciona el talento académico con la relación entre padres e hijos, particularmente el estilo parental (e.g. rígido) y el tipo de relación de apego con los hijos. Asimismo, se ha encontrado que existe una relación entre el perfeccionismo, el grado de las demandas exigidas y el nivel de respuesta hacia los hijos por parte de los padres (Speirs Neumeister \& Finch, 2006).

En complemento, Chan $(2006 ; 2007 ; 2008)$ realizó estudios acerca del perfeccionismo adaptativo y maladaptativo en estudiantes dotados chinos, sentando bases teóricas para la relación directa entre el bienestar subjetivo y el perfeccionismo adaptativo, en contraste con la relación directa entre el perfeccionismo maladaptativo y el malestar subjetivo. Chan también menciona la existencia de signos de multipotencialidad entre los alumnos talentosos, definiéndolo como la tendencia de intentar alcanzar el éxito en la mayor cantidad de áreas posibles, mostrándolo como uno de los principales rasgos de desajuste dentro de la población. Por último, en sus investigaciones deja en claro la necesidad de ahondar en la importancia de las variables socioculturales que inciden en el perfeccionismo dentro de la población de estudiantes académicamente talentosos. 
Speirs Neumeister (2004) llevó a cabo un estudio en base a las dimensiones descritas por Hewitt, Flett, Turnbull-Donovan y Mikail (1991), con el fin de identificar las variables que influenciarían el desarrollo del perfeccionismo en alumnos con alta capacidad en educación básica. Los hallazgos mencionan que un estilo de crianza autoritario y una autovaloración basada en los resultados se relacionan con el perfeccionismo socialmente prescrito de Hewitt et al. (1991), mientras que experiencias tempranas gratificantes, entre otras, se relacionarían con un perfeccionismo orientado a sí mismo.

En relación con los aspectos contextuales involucrados en la temática, es necesario poner en relieve el rol de la dimensión educacional en la interacción entre el talento académico y el perfeccionismo. Por un lado, ciertas prácticas pedagógicas podrían estar en estrecho vínculo con diferentes manifestaciones perfeccionistas, tal como indica Bellamy (1990), quien encontró que factores tales como las expectativas docentes en colegios públicos y privados podrían influir y potenciar tales comportamientos en alumnos.

Más adelante, Freeman (2004) afirma que la mayoría de los desafíos que enfrentan los individuos talentosos provienen de las diversas presiones que el medio pone sobre ellos. Al realizar un estudio comparativo entre jóvenes socialmente etiquetados como talentosos y jóvenes igualmente de alto rendimiento pero no etiquetados oficialmente, señala que los rasgos de perfeccionismo son observados sólo en el primer grupo, lo que sienta bases para hipotetizar la relación entre el perfeccionismo y autoconcepto en el talento académico.

Es importante señalar que existe la necesidad de generar información y nuevos estudios que contribuyan a comprender la manifestación del perfeccionismo en todas sus variaciones, así como de los factores educativos, culturales, familiares, etc., que estarían en relación con la necesidad de alcanzar la excelencia. Se evidencia un vacío teórico existente en cuanto a las implicancias que el perfeccionismo como rasgo de identidad conlleva para los sujetos académicamente talentosos, dentro del contexto situado en Latinoamérica, y a partir de una investigación cualitativa que rescate los significados creados por sus propios protagonistas. 
La actual investigación propone buscar resultados que logren amortiguar esta falta e idealmente comenzar una línea investigativa dentro de la región bajo estos conceptos.

El propósito del presente trabajo consiste en conocer las manifestaciones del perfeccionismo a la luz de la experiencia y los significados construidos por jóvenes académicamente talentosos. En virtud de lo expuesto, la presente investigación plantea las siguientes preguntas de investigación:

1. ¿Cuáles son las vivencias y significados asociados al perfeccionismo por parte de estudiantes con alta capacidad?

2. ¿Cómo influye el medio sociocultural y educativo en la construcción del perfeccionismo en estudiantes con alta capacidad?

3. ¿Cuáles son las principales áreas que los jóvenes participantes del estudio refieren ver afectadas por el perfeccionismo adaptativo y desadaptativo, y de qué forma?

\section{Método}

A partir del cuerpo teórico de la investigación, se concibe al talento académico y al perfeccionismo como constructos devenidos desde y en relación constante con el espacio social, en tanto ambas son etiquetas colectivas en correspondencia con dispositivos sociales y funcionales dentro de un determinado contexto (instituciones educativas, academias científicas, clínica y salud mental, entre otros). Desde lo anterior, la problemática se concibe desde un enfoque fenomenológico, puesto que se busca la comprensión de los significados construidos por los protagonistas, cuya mirada en torno a su realidad concreta se intenta transparentar.

A partir de la revisión bibliográfica acerca del perfeccionismo en estudiantes chilenos académicamente talentosos, se evidencia un vacío teórico importante en la temática, por lo que se define este como un estudio de casos exploratorio, procurando generar una familiarización inicial con el tema de estudio en torno a los contenidos que emerjan desde el relato de los sujetos (Yin, 1989). 
Caracterización del perfeccionismo en estudiantes con alta capacidad / González et al.

\section{Participantes}

\section{Participantes perfeccionismo adaptativo}

-Sebastián, de 15 años, cursa segundo año de enseñanza media (secundaria). Es el mayor de dos hermanos, vive con ambos padres y asegura tener una muy buena relación familiar. Declara que en sus tiempos libres es fundamental practicar diversas actividades deportivas, repasar contenidos académicos y abordar todo tipo de actividades que contribuyan con su crecimiento integral.

-Marcela tiene 17 años al momento de ser entrevistada y cursa cuarto año medio. Es la mayor de dos hermanas y juntas viven con ambos padres; asegura que las dinámicas familiares son de gran calidez y unión. Se define como una alumna meticulosa, aunque solo en aquellas materias de su interés, mientras que presta escasa atención a otras, en las que ha debido incluso trabajar con psicopedagogos y acceder a evaluaciones diferenciales debido a su diagnóstico de discalculia.

\section{Participantes perfeccionismo desadaptativo}

-Sofía es una joven de 17 años que cursa cuarto año medio; también cursa un preuniversitario y un propedéutico como preparación para la universidad. Es hija única y vive con su madre y padrastro. Afirma que hace un tiempo practicó con mucho esmero actividades como fútbol, básquetbol, natación y tae kwon do, pero que debió dejarlos por falta de tiempo y por haber privilegiado participar en actividades académicas.

-Enrique, de 16 años, es el mayor de tres hermanos varones y vive con ambos padres. Refiere que desde que entró a Beta, el año anterior a este estudio, ha sentido la presión de altas expectativas por parte de sus profesores, quienes le exigen un rendimiento sobresaliente. Asegura que fue esto lo que lo llevó a repetir tercer año medio y haber tenido que cambiarse de colegio durante el año en trascurso a un mes de iniciado el año escolar. En cuanto a otras actividades, dice disfrutar de la práctica del karate, así como de tocar guitarra y actualmente explora en la pintura y dibujo debido a que su madre habría descubierto en él habilidades para ello. 


\section{Medición}

En la primera etapa de investigación, y sólo con fines de selección de casos, se administró la Escala Multidimensional de Perfeccionismo (MPS) (Frost, Marten, Lahart \& Ronseblate, 1990), la que se encuentra compuesta por seis subescalas: Preocupación por los Errores (CM); Estándares Personales (PS); Duda sobre las Acciones (DA); Expectativas Parentales (PE); Crítica Parental (PC); y Organización (O). Las subescalas de PS, PE y O muestran alta correlación con un estilo de perfeccionismo adaptativo, mientras que las escalas de CM, DA y PC se correlacionan con la presencia de un perfeccionismo más bien desadaptativo. Un puntaje sobre dos desviaciones estándar por sobre la media fueron consideradas como un puntaje alto para cada una de las categorías. Se ha encontrado un coeficiente de confiabilidad -medido a través de Alfa de Cronbach—entre .85 y .87 para la MPS versión español (Franco, Mancilla-Díaz, Álvarez \& Vázquez, 2010) y coeficientes altos de validez convergente con escalas que miden otros rasgos clínicos (Carrasco, Belloch \& Perpiñá, 2010).

Posteriormente, se realizaron entrevistas en profundidad semiestructuradas a los cuatro seleccionados a partir de los resultados de la escala anteriormente mencionada. El listado de preguntas realizadas a los participantes fue confeccionada a partir de los tópicos de análisis de datos de Kerr, Colangelo y Gaeth (1988) y de adaptaciones del instrumento utilizado por Speirs Neumeister (2004), además de aquellas confeccionadas por las investigadoras para apuntar al contexto sociocultural de los sujetos, resultando así un listado de nueve preguntas que se ilustran en la Tabla 1. Las entrevistas se realizaron en dependencias del programa Beta y tuvieron una duración aproximada de una hora. Dichas entrevistas fueron grabadas en audio para ser posteriormente transcritas para los análisis correspondientes. 


\section{Tabla 1}

Preguntas Entrevista en Profundidad

\section{Preguntas}

1. Qué significa para ti ser un estudiante con alta capacidad?

1.1 Contextualización del perfeccionismo para mejor comprensión del estudiante

2. ¿Te consideras perfeccionista? ¿Por qué?

3. Piensa en alguna vez en que te hayas sorprendido siendo perfeccionista y cuéntame sobre ello con todos los detalles posibles.

4. ¿Cómo es ser perfeccionista?

5. ¿Crees que tus papás tengan que ver con tu perfeccionismo? ¿Por qué?

6. ¿Cómo se involucran ellos en tu desempeño académico?

7. ¿Crees que ser hombre/mujer influya en ser perfeccionista?

8. ¿Crees que tu ambiente escolar tenga que ver con tu perfeccionismo?

9. ¿Qué crees tú que te llevó a ser perfeccionista?

\section{Procedimiento}

La investigación se realizó durante el primer semestre del año 2014 con jóvenes pertenecientes al programa BETA, entidad que trabaja al alero de una universidad chilena ubicada en la región de Valparaíso. Dicho programa está orientado a abordar las necesidades educativas de estudiantes con talento académico de la región, a través de la modalidad de enriquecimiento extracurricular, en donde se brindan oportunidades académicas de alta calidad para alumnos(as) provenientes de escuelas y liceos municipales (públicos) entre 12 y 18 años, quienes asisten a cursos y talleres impartidos durante todo el ańo académico (viernes y sábado) más una temporada de verano (enero).

Se realizó el contacto inicial para este estudio con representantes del programa Beta-PUCV, quienes accedieron a participar de la investigación, realizando el vínculo entre las investigadoras y los alumnos. Posteriormente, se realizó la aplicación de la Escala Multidimensional de Perfeccionismo (Frost, Marten \& Lahart, 1990), de forma colectiva 
a 133 estudiantes asistentes al programa Beta-PUCV de entre 15 y 19 años con el fin de detectar puntajes extremos en cuanto a la variable de perfeccionismo y poder conformar la muestra de estudio. De esta manera, la escala es utilizada sólo con fines instrumentales para encontrar los casos, siguiendo los procedimientos del estudio realizado por Neumeister (2004) focalizado en el estudio del perfeccionismo en estudiantes talentosos universitarios. A continuación, en una vertiente cualitativa, se realiza un muestreo propositivo procurando mantener en proporciones homogéneas la variable de género y edad. Se seleccionaron como participantes a cuatro jóvenes, dos integrantes para cada categoría de la escala de perfeccionismo (adaptativo y desadaptativo), un participante de cada género para ambas escalas, siendo estos aquellos que tuviesen el mayor puntaje de su clasificación. La totalidad de la investigación fue realizada bajo la supervisión de un equipo multidisciplinario a cargo del programa, con aplicación de consentimientos informados y acuerdos de confidencialidad para los padres y alumnos. Por lo anterior, los participantes serán identificados con nombres ficticios, mientras que se adjuntan datos biográficos generales y no específicos para una mejor comprensión de los casos en cuestión.

\section{Análisis de los datos}

La información recabada desde las entrevistas en profundidad previamente transcritas fue sometida a un análisis inductivo en profundidad a la luz de la teoría existente (Strauss \& Corbin, 1990). En cuanto a los procedimientos de análisis, se realizó: (a) lectura y relectura de las transcripciones, (b) organización de datos a través de códigos, (c) comparación constante entre códigos y categorías y la literatura relevante y (d) establecimiento de relaciones entre categorías.

El análisis de las unidades de información se realizó mediante contenido categorial temático (Cabrera, 2013), con el fin de descomponer el texto en unidades para después ir agrupando en categorías de acuerdo a similitudes y, de esta forma, extraer de forma metódica los significados construidos acerca del perfeccionismo desde el material 
discursivo entregado por los estudiantes académicamente talentosos. Se realizaron codificaciones sistemáticas y exhaustivas del material, a modo de encontrar aquellos pasajes relevantes para dar respuesta a las preguntas de investigación. Posteriormente, los significados emanados de los discursos de los estudiantes fueron categorizados procurando siempre elaborar categorías en niveles altos de abstracción (Schreier, 2014).

\section{Resultados}

Una vez seleccionados los alumnos para cada tipo de perfeccionismo, se comenzó con la fase cualitativa del estudio: los resultados de tal etapa son expuestos de forma comparativa y contrastando ambos tipos de perfeccionismo, considerando que los participantes fueron seleccionados por sus puntajes extremos para ambas tipologías.

A partir del análisis de contenido de las entrevistas en profundidad, se encontraron puntos en común entre todos los entrevistados, como su propio reconocimiento como perfeccionistas, la reflexión constante que realizan acerca de su rasgo de personalidad, los que refieren haber notado con el inicio de la etapa preescolar y que atribuyen a factores ambientales específicos.

Con respecto a dichos factores, así como las áreas en las que el perfeccionismo muestra influencia, los jóvenes coinciden en su identificación, pero difieren en la forma en que estas han sido vivenciadas. A partir de las diferencias en cuanto a la vivencia propiamente tal del perfeccionismo a través de su historia personal, se muestran a continuación los resultados separados para perfeccionistas adaptativos y desadaptativos. Para cada grupo se presentan cuatro categorías transversales en donde ambas manifestaciones del perfeccionismo muestran divergencias nucleares: se observa la importancia de la influencia del núcleo familiar a través de la transmisión de diferentes manifestaciones perfeccionistas o creencias en torno a la excelencia. Asimismo, a pesar de que todos los participantes mantenían plena conciencia acerca 
de sus rasgos perfeccionistas y han realizado ocasionales reflexiones acerca de ello con anterioridad, ambos grupos muestran tendencias opuestas en torno a la vivencia del perfeccionismo como fenómeno identitario, destacando el aspecto experiencial, la significación del concepto y las diferentes estrategias que se establecen para llevar los impulsos perfeccionistas al acto. Todo lo anterior conlleva repercusiones en la dimensión social del sujeto perfeccionista, mientras que el relato acerca del contexto socioeducativo aparece constantemente a lo largo de la narrativa. Una síntesis de los resultados en cada categoría y a través de los casos se presenta en la tabla 2.

\section{Tabla 2}

Principales resultados por categorias a través de los casos de la muestra

\begin{tabular}{|c|c|c|c|c|c|}
\hline & Casos & $\begin{array}{l}\text { Influencia del } \\
\text { núcleo familiar }\end{array}$ & $\begin{array}{l}\text { Vivencia del } \\
\text { perfeccionismo }\end{array}$ & $\begin{array}{l}\text { Contexto } \\
\text { social }\end{array}$ & $\begin{array}{l}\text { Ámbito } \\
\text { educativo }\end{array}$ \\
\hline $\begin{array}{l}\text { Perfec- } \\
\text { cionismo } \\
\text { adaptativo }\end{array}$ & $\begin{array}{l}\text { Sebastián } \\
\text { Marcela }\end{array}$ & $\begin{array}{l}\text { - Valoración } \\
\text { positiva del } \\
\text { éxito } \\
\text {-Involucra- } \\
\text { miento sin } \\
\text { exigencia exce- } \\
\text { siva }\end{array}$ & $\begin{array}{l}\text { - Satisfacción } \\
\text { hacia el esfuerzo } \\
\text { - Error como } \\
\text { fuente de } \\
\text { aprendizaje } \\
\text { - Motivación } \\
\text { intrínseca hacia } \\
\text { áreas de interés }\end{array}$ & $\begin{array}{l}\text { - Desestima- } \\
\text { ción de lo } \\
\text { negativo y } \\
\text { apropiación } \\
\text { de experien- } \\
\text { cias positivas } \\
\text { - Liderazgo }\end{array}$ & $\begin{array}{l}\text { Visión } \\
\text { instrumental }\end{array}$ \\
\hline $\begin{array}{l}\text { Perfec- } \\
\text { cionismo } \\
\text { desadap- } \\
\text { tativo }\end{array}$ & $\begin{array}{l}\text { Enrique } \\
\text { Sofía }\end{array}$ & $\begin{array}{l}\text { - Exigencia } \\
\text { continua } \\
\text { - Involu- } \\
\text { cramiento } \\
\text { presencial de } \\
\text { los padres en } \\
\text { actividades de } \\
\text { los hijos }\end{array}$ & $\begin{array}{l}\text { - Multicapa- } \\
\text { cidad } \\
\text { - Autoexigencia } \\
\text { con fines } \\
\text { extrínsecos o } \\
\text { instrumentales }\end{array}$ & $\begin{array}{l}\text { - Alejamiento } \\
\text { de los pares }\end{array}$ & $\begin{array}{l}\text { Presión } \\
\text { del medio } \\
\text { educativo } \\
\text { por cumplir } \\
\text { ciertos están- } \\
\text { dares }\end{array}$ \\
\hline
\end{tabular}


Caracterización del perfeccionismo en estudiantes con alta capacidad / González et al.

\section{Perfeccionismo adaptativo}

Influencia del núcleo familiar. Dicha influencia en el perfeccionismo adaptativo es plasmada en las entrevistas a través de la referencia a padres responsivos que entregan un apoyo incondicional e independiente de los resultados obtenidos por sus hijos. Dejan, además, lugar para altos niveles de autonomía y libertad de elección en cuanto a las áreas que sus hijos deseen explorar, sin ejercer mayor presión e interviniendo solo en la medida y momento en que es requerido.

Además, se promueven modelos parentales que fomentan una actitud perfeccionista basada en el disfrute de tareas específicas y su realización en la convivencia cotidiana dentro del grupo familiar, enfatizando la enseñanza de padres a hijos acerca de cómo construir patrones perfeccionistas saludables:

Por ejemplo, nosotras, cuando éramos muy niñas, y tal vez tenga que ver, ayudábamos a pulir cosas, porque en mi casa hay como un anticuario, entonces habia que pulir muchas cosas de bronce, manijas de puertas, (...) entonces el trabajo nuestro a los ocho años tenía que ser súper riguroso, no podía quedar feo, no podíamos rayarlo, no podíamos botarlo y doblarlo, no eran cosas que se podian hacer. Entonces yo creo que el colaborar constantemente con los trabajos de nuestros papás en aquellas cosas que eran más pequeñas, que no se podian hacer como bruto, yo creo que ayudó también a que fuéramos cuidadosos. (Marcela)

Agrega luego: (Mis padres) siempre están interesados en que les contemos con mi hermana qué hicimos, qué cursos tomamos, pero nunca, nunca nos han puesto limitaciones (...) nunca me han puesto problemas con respecto a si rindo poco (...), nunca han sido para mi una presión. Mis papás nunca me exigieron ser una persona ejemplar aparte de ser una persona decente desde el punto de vista moral. Nunca me exigieron ser buena en todo, yo tampoco me exijo ser buena en todo. Mi familia me apoyó, el colegio no tanto, pero mi familia sí y con eso me bastó.

Sobre lo mismo, Sebastián comenta: mis papás siempre me han felicitado porque no necesito ayuda de ellos o un apoyo o empujón para tratar de ser mejor en las notas y en las capacidades, porque siempre yo mismo me 
pongo las metas y las cumplo. Cuando yo tengo un logro, ellos tratan de felicitarme y valorar eso para que yo siga siendo mejor.

Vivencia del perfeccionismo. El perfeccionista adaptativo se caracteriza por realizar una reflexión, reconocimiento y evaluación consciente acerca de las propias aptitudes y capacidades: $O$ me preocupaba de ser una niña promedio o me preocupaba de mejorar aquellas cosas en las que podía ser excelente. Entonces podría lograr tener un 4.0 en matemáticas o podría preocuparme y gastar mi tiempo en cantar, estudiar técnica lírica, viajar o escribir, bordar, o preocuparme de estudiar tres veces a la semana una materia que no me gusta, en la que no soy buena y en la que mi mayor expectativa era sacarme un 4.0. Entonces me dejó de importar. Decidi que no me importara. (Marcela)

Al existir mayores niveles de independencia en cuanto a los intereses personales, es coherente que destaquen como factor motivacional para buscar la excelencia a sus propias preferencias y compromisos afectivos, concentrando todos sus esfuerzos principalmente en áreas específicas de su preferencia, que les apasionen y que rindan frutos atractivos para ellos. Marcela declara: Empecé a darme cuenta de que las cosas en las que me estaba preocupando de ser buena, me gustaban. Me gustaba hacerlas, amaba hacerlas, la pasaba bien haciéndolas y me distraian de otras que no me gustaban tanto.

Asimismo, esta dinámica se transforma incluso en un factor protector ante las vicisitudes del medio y de la resolución de tareas: No es que me guste estudiar, sino que me gusta aprender de cosas que a mi me gustan, cuando son cosas para el colegio, siendo súper sincera, no me estreso de gran forma. Me estreso con las cosas que son mias. (Marcela)

En torno a lo anterior, las estrategias adoptadas por el perfeccionista adaptativo para llevar al acto sus intenciones de conseguir la excelencia se basan en sentar límites saludables para el esfuerzo, diferenciando cuándo y dónde prefieren focalizar sus energías y detectando agentes nocivos para su bienestar: Casi siempre logro mis metas, y si no las logro me las propongo para un tiempo más y trato de lograrlas ahí." "Yo lo he comprobado a lo largo de vida, no se necesita ser tan perfeccionista en todo. (Marcela) 
Lo anterior promueve índices de bienestar subjetivo, pues el perfeccionismo suele ser asumido como rasgo identitario que conlleva un disfrute y placer en la práctica de las tareas escogidas. Al preguntarle acerca de las implicancias del perfeccionismo en su vida, Marcela dice: Es una característica mía creo. Creo que ha hecho que desarrolle cosas positivas.

Se destaca la gratificación obtenida del logro en sí mismo luego de alcanzar una meta esperada. A su juicio, esto conlleva un desarrollo humano, mientras que poseen una visión más positiva acerca del proceso de aprendizaje y de su etiqueta de talentos académicos: $\mathrm{Me}$ encanta esforzarme por algo. No me gusta que las cosas sean fáciles, me gusta esforzarme por las cosas, dando todo de mí. Me hace sentir, de alguna forma, feliz. (Sebastián)

Al mismo tiempo, los errores son considerados como oportunidades para realizar análisis metacognitivos y reflexivos, considerándolos como oportunidades para reformular el actual desempeño. Sobre este tema, Sebastián remarca: Hay que tener errores para aprender. A veces, antes de acostarme, pienso en algún error o alguna mala palabra que pueda haberle caido mal a alguna persona o errores pequeños que haya cometido para no volver a cometerlos al otro día. Un proceso de ver todo lo que uno ha hecho en el día.

Dimensión social. El perfeccionista adaptativo muestra una convivencia gratificante con sus pares, principalmente porque, como ellos enfatizan, han aprendido a sortear los obstáculos que las dinámicas escolares han interpuesto en sus procesos de socialización al desestimar las relaciones nocivas y conservar las nutritivas. Al consultar a Sebastián si el perfeccionismo conlleva consecuencias negativas socialmente, responde: no, porque las cosas negativas trato de sobrepasarlas o superarlas, como subir las notas o los malos comentarios. Evitarlos no más.

Además de esto, se evidencian rasgos éticos y morales asociados con una tendencia altruista marcada, que se manifiesta en la necesidad de realizar actividades relacionadas con el bienestar social, asumir roles de liderazgo, colaboración entre pares, etc.: Si yo soy feliz puedo ayudarlos, ayudarlos a que ellos también sean mejores. Yo soy ese típico que está ahi, que no se burla de los compañeros, que no hace eso y después va y 
le dice 'yo te apoyo, yo pienso lo mismo que tú'. Me gusta ayudar también a la gente. (Sebastián)

Contexto socioeducativo. En general todos los estudiantes de la muestra hacen referencia a prácticas pedagógicas poco inclusivas. Sin embargo, los jóvenes perfeccionistas adaptativos son enfáticos en expresar su apreciación instrumental hacia las calificaciones en sus respectivos colegios, aclarando que sólo se esfuerzan de manera importante en aquellos ramos de su preferencia, mientras que en el resto solo se desempeñan adecuadamente para obtener un buen promedio o satisfacer a sus padres, generando esto calificaciones de rango heterogéneo: Francamente [las notas] no me importan mucho. Me preocupan porque sé que las tengo que tener en algún momento, y sé que tengo la presión del NEM4. Si no fuera por eso en verdad no me importarian mucho. Siendo sincera, no me importa mucho si cumpli o no con el reglamento interno a cabalidad con respecto a mi comportamiento, mi asistencia, mi puntualidad y esas cosas. No se convierten en algo relevante en mi vida. Es instrumental para mí. (Marcela)

\section{Perfeccionismo desadaptativo}

En comparación con lo anterior, el perfeccionismo desadaptativo muestra rasgos casi completamente opuestos en torno a las categorías de análisis revisadas.

Influencia del núcleo familiar. En el perfeccionismo desadaptativo, los padres de estos jóvenes cumplen una función de mayor control y presión constantes, instaurando la idea de que el rendimiento excelente es primordial y que debe ser transversal a cada área abarcada por un sujeto de altas capacidades. La idea de que la excelencia se relaciona con instancias de superación ante la adversidad es potenciada a través del ejemplo parental, discurso que los alumnos hacen propio y toman como referencia para el propio desempeño como una herencia inmaterial: La superación de mis papás influyó en un porcentaje no menor. Porque

4 NEM se refiere a Notas de Enseñanza Media, el conjunto de calificaciones obtenidas en los 4 años de enseńanza secundaria y que influyen en el puntaje de ingreso a la educación superior. 
Caracterización del perfeccionismo en estudiantes con alta capacidad / González et al.

si veo que ellos pudieron, ¿por qué yo no? Porque si ellos pudieron en esa época, con esos recursos, ¿por qué yo no puedo con los recursos que tengo, y con las capacidades que ellos me otorgaron a mi? ¿Por qué no? (Enrique).

También los padres de perfeccionistas desadaptativos promueven la idea de capacidades ilimitadas en sus hijos, la que tales adolescentes practican tratando de conseguir resultados destacados en diversas áreas que muchas veces son impuestas por ellos, excediendo en ocasiones los límites saludables e incluso inhibiendo el rendimiento académico: Estuve un tiempo pensando que no podría seguir aqui (Beta) porque tenia que hacer muchas cosas, pero mis papás me decian que no, que esto me servia. Siempre me dicen que siga, que siga no más, que siempre tenia que ser la mejor. Siempre me andan diciendo que estudie, porque al fin eso es lo unico que ellos me pueden dejar en concreto. Que estudie para ser alguien mejor. Siempre insistían que estudiara, que estudiara, que estudiara. Mi padrastro es muy perfeccionista. Yo creo que eso me llevó a ser así. (Sofía)

Se observa que los estudiantes perfeccionistas desadaptativos utilizan sus capacidades y rendimientos como una forma de enaltecimiento de los padres en una dinámica en donde cuyas indicaciones no son cuestionadas: A mi mamá no le gusta que yo practique fútbol. Dice que no me va a llevar a nada. Este año tuve que dejar fútbol por asistir al preuniversitario, al propedéutico y a Beta. Entonces, dejo de lado lo que me gusta por seguir los estudios y tratar de seguir adelante, ser la mejor. (Sofía)

Vivencia del perfeccionismo. A diferencia de los entrevistados para el perfeccionismo adaptativo, los sujetos comprendidos en la categoría de perfeccionismo desadaptativo suelen sacrificar sus preferencias acerca de en qué áreas específicas desean desempeñarse en relación con sus propias capacidades, mientras que se observa la necesidad de abarcar la mayor cantidad posible de actividades, existiendo una sensación de multicapacidad: Desde que iba en Kinder me decían 'tú tienes un potencial ilimitado' y entonces, hace poco tiempo me empecé a dar cuenta de eso y me empecé a explotar. Y los fui explotando, los fui explotando, los fui explotando, hasta que llegó un punto donde no los pude explotar más y de ahi los segui explotando hasta romper esa barrera que me decía 'tú no puedes'. $Y$ ahora me di cuenta de que nada me va a detener (rie) (Enrique). 
Así mismo, los entrevistados sostienen que la principal motivación para alcanzar el éxito proviene de la necesidad de conseguir la aprobación externa, de comprobar la capacidad de alcanzar los estándares de rendimiento — reales o hipotéticos- del medio, junto con la autoimposición de conseguir la excelencia como un deber con fines instrumentales, como la entrada a la universidad o una estabilidad económica superior: No me gusta que los demás me miren, no me gustaria que los demás me miraran y me dijeran 'a ella le va mal en todo, no se esfuerza'. Siento que puedo y también siento que debo demostrarle a los demás que puedo. (Sofía)

En el caso específico de Enrique, la autoexigencia tuvo consecuencias aún más complejas para su bienestar subjetivo, involucrando un detrimento de su salud mental: (El perfeccionismo) me generó mucho stress, a un punto muy alto. Hace poco tuve un problema. Esa fue la gota que derramó el vaso. Tuve un problema de stress muy fuerte. Es algo un poco complicado.

En cuanto a la consideración de los errores, existe un rechazo hacia estos, pues son vistos como un fracaso y conlleva una carga que cobra valor en un malestar propio a causa de que este malestar se extiende hacia figuras significativas; mientras el éxito es motivo de valoración positiva por parte de estas, los errores representan una amenaza que pondría en riesgo el bienestar de padres y pares: Si fracasara, mis padres pensarian por qué está pasando, qué es lo que está pasando, que quizás ellos han hecho algo mal. Se sentirian culpables porque dirian 'iqué hemos hecho mal?'. A veces eso pasa, que bajo las notas y me dicen '¿qué pasó? ¿por qué estás asi?' (Sofía)

En consecuencia con lo anterior, dentro del perfeccionismo desadaptativo se evidencia el sacrificio del propio bienestar a causa de estrategias rígidas y poco gratificantes, menor tolerancia al fracaso y límites difusos en cuanto a las propias capacidades, lo que lleva en ocasiones a la extralimitación, stress y agotamiento severo.

Dimensión social. Se observan ciertas dificultades debido a características de personalidad específicas y relacionadas con la baja tolerancia a la frustración. Enrique dice: $A$ veces me aleja de las personas, me enojo 
conmigo y dejo cosas de lado. A esto, se suma que dichos sujetos suelen invertir mayor cantidad de tiempo en obtener resultados cada vez mejores antes que socializar con sus pares. En adición, se observa la gestión de relaciones poco espontáneas, basadas en el intercambio de conocimiento y fines instrumentales como estrategia para obtener más aceptación social y satisfacer necesidades de compañía.

Contexto socioeducativo. Los jóvenes de este grupo declaran haber sentido gran presión ejercida por sus profesores en torno a las calificaciones y el cumplimiento del reglamento escolar, llevando incluso al efecto contraproducente de la merma del rendimiento: Me decían 'Usted es un alumno Beta, tiene que tener un promedio perfecto'. Siempre me han cargado con eso, me ha hecho sentir algo presionado, porque si no doy lo mejor de mí, o si llego a dar lo mejor de mi y no es suficiente, los voy a decepcionar. Y eso es algo que me frenaba a dar mi $100 \%$ en lo que tenía que ver con el colegio. (Enrique)

El espacio escolar es percibido entonces como un lugar de cuestionable acogida para tales jóvenes, y más aún como un vehículo para obtener el puntaje de calificaciones suficientes como para alcanzar metas posteriores, de acuerdo a las exigencias de sus padres y de la sociedad, y principalmente para garantizar la movilidad social.

\section{Discusión}

En términos generales y a través del discurso de los entrevistados, se dejan ver los efectos que para ellos conlleva la etiqueta del talento académico sumada a su condición de sujetos perfeccionistas, generando diversas consecuencias que van desde el aislamiento social, dificultad para establecer relaciones satisfactorias con pares, rechazo por parte del entorno (Colangelo, 2002; Freeman, 2000a; Robinson, 2008), hasta la inhibición del rendimiento académico y bajas en el autoestima, eventos

que los sujetos entrevistados narran con variable compromiso afectivo y disposición hacia el contexto. De acuerdo con esto, cobra sentido lo afirmado por Freeman (1998) en cuanto al peso que la sociedad pone 
sobre estos jóvenes a través de etiquetas sociales que promueven estereotipos y roles sociales rígidos y encapsulantes.

Es esta subjetividad con la que los estudiantes entrevistados hacen referencia al contexto social, lo que resulta más llamativo dentro del análisis y que marca las principales diferencias entre los rasgos del perfeccionismo adaptativo y desadaptativo. La caracterización del perfeccionismo tanto adaptativo como desadaptativo será expuesta a continuación.

Realizando una síntesis, los entrevistados en torno al perfeccionismo desadaptativo expresaron una consideración del entorno como un espacio de demostración de la propia multipotencialidad, donde las capacidades individuales se configuran como herramientas para obtener recompensas asociadas a la movilidad social y económica, y el reconocimiento social. Tal idea es reforzada por los grupos familiares, quienes además de entregar a sus hijos la máxima cantidad de oportunidades pedagógicas como herencia y patrimonio inmaterial, se encargan de transmitir sus propias narraciones de esfuerzo y superación como indicaciones incuestionables, ejerciendo así gran presión a través de altas expectativas parentales, mayores exigencias y una supervisión más cercana y constante sobre lo que realizan sus hijos(as), lo cual se condice con lo encontrado por Speirs Neumeister y Finch (2006) sobre la influencia de los estilos parentales en el perfeccionismo desadaptativo. Acerca de ello, recordamos que los entrevistados relacionan tales presiones con mermas en su rendimiento debido al miedo a fracasar; es decir, altas expectativas parentales no siempre se relacionarían con la excelencia académica (González, Leal, Segovia \& Arancibia, 2012).

A lo anterior se agrega la referencia a un sistema educativo que no siempre atiende estas necesidades, con expectativas docentes estereotipadas que contribuyen a presionar a los alumnos con similitud a las expectativas parentales (Bellamy, 1990), sin ofrecer la suficiente contención ni herramientas para lograr un proceso pedagógico de calidad, creando un círculo vicioso en el que el alumno con talento académico podría quedar atrapado por las exigencias externas (Hewitt et al., 1991). Incluso, los perfeccionistas desadaptativos entrevistados 
sugieren que el contexto escolar se convierte en un dispositivo que condiciona la propia valía. En este sentido, cabe destacar que las altas expectativas externas encontradas en el perfeccionismo maladaptativo, tal como lo evidenciaron los entrevistados, se basan en un sustrato efectivamente real y basado en un sistema social que reforzaría esta idea.

En contraste, los sujetos perfeccionistas adaptativos mostraron una visión más próspera del entorno, considerándolo como una fuente inagotable de experiencias enriquecedoras que contribuyen con su desarrollo personal. La sociedad en sí es vista como un espacio de crecimiento, donde se genera un aprendizaje significativo y colaborativo en función del bien comunitario, lo que se vincula directamente con los rasgos de liderazgo y empatía narrados por tales jóvenes, en concordancia con la visión acerca del entorno y satisfacción de vida propuesta por Stoeber y Rambow (2007) para los perfeccionistas adaptativos.

Los padres de los alumnos entrevistados e identificados como perfeccionistas adaptativos han reforzado dicha idea, generando un ambiente seguro y protegido para la exploración libre de áreas de su preferencia. Esta idea propone una teorización distinta a lo expuesto por Colangelo (2002) quien identifica patrones generalizados de estilos relacionales coercitivos en familias de alumnos talentosos, mientras que los hallazgos coinciden con investigaciones anteriores que destacan el núcleo familiar como central en el origen del perfeccionismo (Chan, 2008; Soenens et al., 2005; Stöeber, 2014). Sobre aquello, es necesario destacar los resultados de Speirs Neumeister (2004) acerca de los factores influyentes en el desarrollo del perfeccionismo: estilos parentales más acogedores, comprensivos y flexibles llevarían al desarrollo de un perfeccionismo orientado a sí mismo — análogo al perfeccionismo adaptativo descrito por los entrevistados de este estudio-, mientras que estilos parentales rígidos, demandantes y poco comprensivos llevarían a un perfeccionismo socialmente prescrito, coincidente con el perfeccionismo desadaptativo examinado en la presente investigación.

En cuanto al contexto escolar, los jóvenes perfeccionistas adaptativos lo consideran solo como un preámbulo para metas superiores que satisfagan sus deseos y necesidades, sabiendo discernir entre 
las iniciativas que en el se den y que realmente les nutra, como amistades, aprendizajes específicos, etc. En estos términos, y respaldado por los dichos de Chan (2008) acerca de las diferentes consecuencias de las dos principales manifestaciones del perfeccionismo, los alumnos perfeccionistas adaptativos entrevistados, al tener una visión más crítica acerca del contexto, se mostraron capaces de discernir entre los estímulos beneficiosos de los nocivos que este les entrega, logrando así una salida socialmente ajustada y mentalmente saludable en términos de bienestar subjetivo, lo que muestra la diferencia fundamental entre ambas manifestaciones del rasgo de personalidad. Así, los sujetos de nuestra investigación refieren que altos niveles de autonomía en diversas áreas, un notable desarrollo moral y contextos familiares que favorezcan ambos factores, se relacionan con un perfeccionismo adaptativo, orientado a sí mismo, que se constituye como un agente beneficioso para el desarrollo humano, bajo los significados construidos por el mismo sujeto.

Al contrario, los jóvenes entrevistados para perfeccionismo desadaptativo, muestran actitudes sumisas ante las condiciones que el contexto social supone, la que los lleva básicamente a sucumbir ante la presión externa, sacrificando sus preferencias personales (Bellamy, 1990; Hamachek, 1978). Es decir, altos niveles de dependencia parental, altas expectativas parentales, potenciado esto por sistemas familiares que promueven la idea de multipotencialidad en sus hijos, una visión adversa del entorno y contextos escolares con expectativas docentes sobredimensionadas, se relacionarían con un perfeccionismo desadaptativo, socialmente prescrito y a merced de satisfacer expectativas y metas impuestas externamente y en base a significados ajenos al sujeto. Cabe aquí destacar lo mencionado por Kreger (1999), en tanto define al perfeccionismo como motor de consecuencias tanto beneficiosas como nocivas para el sujeto dependiendo de los significados que este cree sobre su condición.

Es importante mencionar que por ser este un primer acercamiento a la temática del perfeccionismo en estudiantes chilenos con alta capacidad, los resultados son más bien incipientes y de carácter 
exploratorio. Asimismo, al tratarse de un estudio de casos, no siempre es posible transferir lo encontrado a la población general. De esta forma, las inferencias son más bien de carácter clínico, lo cual permite una aproximación a la manifestación del fenómeno, una generalización intra-casos de modo de poder situarlos y comprenderlos en profundidad (Geertz, 1987). Otra limitación del estudio es su relación con el análisis, ya que sólo dos personas fueron las encargadas de codificar y categorizar los datos producidos, faltando probablemente un tercero para un análisis triangulado.

Finalmente, se puede señalar que los resultados generales de la presente investigación se condicen altamente con aquellos alcanzados en la investigación de Speirs Neumeister (2004): existiría una marcada relación entre el perfeccionismo adaptativo (orientado a sí mismo) y conductas saludables y enriquecedoras en el área familiar, social y pedagógica, mientras que el perfeccionismo desadaptativo (prescrito socialmente) se relaciona, entre otros, con la propia valía condicionada a los resultados y presión ante las expectativas externas.

Si bien este estudio se ha configurado como una investigación exploratoria acerca del perfeccionismo en estudiantes académicamente talentosos en Chile, también es del interés de los investigadores generar información que contribuya a sentar bases para la posible revisión de las prácticas clínicas y pedagógicas en torno al trabajo con estudiantes destacados.

Es necesario recordar que el estudio en profundidad del perfeccionismo como constructo multidimensional se ha situado principalmente en países anglosajones, donde la realidad educativa y social se define en torno a principios bastante distintos a los chilenos. De acuerdo con ello y con la posición paradigmática que se ha adoptado, los significados construidos por alumnos académicamente talentosos que mostraron índices de perfeccionismo fueron rescatados, generando así información atingente con una realidad específica y situada dentro del contexto social chileno.

En términos generales, los hallazgos de este estudio acerca de los aspectos culturales del perfeccionismo indicarían que existe una doble 
significación hacia la perfección. Por un lado, esta es vista como un fenómeno específico y de poca frecuencia, instancia en la que es reconocida y premiada por la sociedad y sus estructuras con diversos incentivos. Sin embargo, la perfección dentro del contexto chileno también conlleva un costo, en tanto los entrevistados expresaron que existiría un precio social para los sujetos que intentan obtener resultados de excelencia, sintiendo la relegación por parte del sistema educativo.

Según lo anterior, si bien la presente investigación considera como definición de lo adaptativo y desadaptativo los índices existentes de bienestar o malestar subjetivo respectivamente definidos por Hamachek (1978), en un análisis más profundo podemos vislumbrar que la adaptación o su contraparte dependerán de la dinámica que se produzca entre la eficacia de las herramientas que los sujetos perfeccionistas posean para lidiar con tales dificultades, y la intensidad con que, en cada caso, el contexto sociocultural inmediato del sujeto haga sentir su presión. Lo anterior marcaría la diferencia entre ambas manifestaciones del concepto en cuestión, existiendo una manifestación adaptativa y otra desadaptativa para el perfeccionismo.

Quizás lo más propio de destacar en cuanto a los resultados de la investigación, es aquello concerniente a los significados y códigos que dan sentido y base al perfeccionismo. Se evidencia a través de la narración de los sujetos que, mientras en el perfeccionismo adaptativo la concepción del éxito se construye a partir de significados propios y de algún modo coherentes con el discurso de los jóvenes, en el perfeccionismo desadaptativo el éxito es comprendido como un sacrificio, una postergación del crecimiento propio a merced de exigencias externas, lo que causa finalmente un deterioro del bienestar subjetivo.

Es pertinente señalar cómo los resultados internacionales acerca de la materia coinciden en su gran mayoría con aquellos obtenidos durante la presente investigación. Sin embargo, para futuras consideraciones es recomendable realizar nuevos estudios que repliquen la investigación en grupos de investigación más amplios y diversos, con el fin de generar información que permita realizar inferencias generalizables a gran parte de la población académicamente talentosa. También resulta interesante 
para la investigación en el área, proponer un estudio comparativo entre poblaciones perfeccionistas etiquetadas y no etiquetadas como académicamente talentosas, con el fin de observar la posible influencia de la etiqueta social del talento en la necesidad de alcanzar la excelencia.

Por otro lado, se evidencia la necesidad de potenciar en nuestro país el trabajo tanto académico como práctico en torno a la población de alumnos doblemente etiquetados como talentosos y perfeccionistas, con el fin de potenciar aquellas tendencias que signifiquen un beneficio para su salud mental y desarrollo académico:

-Con el fin de propiciar patrones de perfeccionismo adaptativo en cambio de aquellos desadaptativos, es necesario masificar el trabajo clínico de apoyo con los alumnos, con el fin de entregar la contención necesaria para lidiar con las posibles vicisitudes que el contexto social pueda anteponer en su desarrollo. También se recomienda potenciar niveles de autoeficacia como herramienta para lidiar con rasgos menos adaptativos inherentes al perfeccionismo en alumnos talentosos (Chan, 2007).

-Es de ayuda también el trabajo clínico con los padres de estos estudiantes, con el fin de reexaminar los patrones de crianza y expectativas parentales y la forma en que estas influyen en el desarrollo de los jóvenes.

También resulta necesario realizar un trabajo colaborativo dentro de los espacios escolares que acogen a alumnos académicamente talentosos, en torno a las prácticas y expectativas docentes y la existencia de programas de enriquecimiento insertos directamente los establecimientos educativos tradicionales, que entreguen las herramientas necesarias a dicha población. 


\section{Referencias bibliográficas}

Adler, A. (1956). The neurotic disposition. In H. L. Ansbacher \& R. R. Ansbacher (Eds.), The individual psychology of Alfred Adler (pp. 239-262). New York: Harper.

Arancibia, V. (2009). La educación de alumnos con talentos: una deuda y una oportunidad para Chile. Santiago de Chile: Pontificia Universidad Católica de Chile. Recuperado de http://www.pentauc. cl/wp/wp-content/uploads/2009/12/Doc-Pol\%C3\%ADticasP\%C3\%BAblicas-Talento.pdf

Bellamy, J. (1990). Perfectionism in adolescents: A comparison of private and public school students. (Tesis de Máster). Universidad de Manitoba, Canadá. Recuperado de mspace.lib.umanitoba.ca/ bitstream/1993/17039/1/Bellamy_Perfectionism_in.pdf

Burns, D. D. (1980). The perfectionist's script for self-defeat. Psychology Today, 34-52.

Cabrera, I. (2013). El análisis de contenido en la investigación educativa: propuesta de fases y procedimientos para la etapa de evaluación de la información. Pedagogía Universitaria, 14(3), 71-93.

Carrasco, A., Belloch, A. y Perpiñá, C. (2010). La evaluación del perfeccionismo: utilidad de la Escala Multidimensional de Perfeccionismo en población española. Análisis y Modificación de Conducta, 2009, 35, 49-65.

Chan, D. (2006). Adjustment problems, self-efficacy, and psychological distress among Chinese gifted students in Hong Kong. Roeper Review, 28(4), 203-209. https://doi.org/10.1080/02783 190609554365

Chan, D. (2007). Positive and Negative Perfectionism among Chinese Gifted Students in Hong Kong: Their Relationships to General Self-Efficacy and Subjective Well-Being. Journal for the Education of the Gifted. 31(1), 77-102. Recuperado de http://files.eric. ed.gov/fulltext/EJ777787.pdf

Chan, D. (2008). Perfectionism and the Striving for Excellence. Educational Research Journal, 23(1). Recuperado de http:// 
hkier.fed.cuhk.edu.hk/journal/wp-content/uploads/2010/06/ erj_v23n1_1-19.pdf

Christopher, M. \& Shewmaker, J. (2010). The Relationship of Perfectionism to affective variables in gifted and highly able children. Gifted child today, 33(3). Recuperado de http://files.eric. ed.gov/fulltext/EJ893803.pdf. https://doi.org/10.1177/107621 751003300307

Colangelo, N. (2002). Counseling Gifted and Talented Students. National Research Center on the Gifted and Talented, Storrs, CT Office of Educational Research and Improvement (ED), Washington, DC. Recuperado de http://www.gifted.uconn. edu/nrcgt/newsletter/fall02/fall022.html

Colangelo, N., Assouline, S. G. \& Gross, M. U. M. (Eds.) (2004). A nation deceived: How schools hold back America's brightest students. Iowa City: University of Iowa. Recuperado de http:// www.accelerationinstitute.org/nation_deceived/nd_v1.pdf

Decreto $\mathrm{N}^{\circ} 170$ (2009). Fija normas para determinar los alumnos con necesidades educativas especiales que serán beneficiarios de las subvenciones para educación especial. Ministerio de Educación de Chile.

Dunkley, D., Zuroff, D. \& Blankstein, K. (2003). Self-Critical Perfectionism and Daily Affect: Dispositional and Situational Influences on Stress and Coping. Journal of Personality and Social Psychology, 84(1), 234-252. Recuperado de http://users. ugent.be/ - wbeyers/scripties2011/artikels/Dunkley\%20et\%20 al._2003.pdf. https://doi.org/10.1037/0022-3514.84.1.234

Ellis, A. \& McLaren, C. (2004). Las relaciones con los demás: terapia del comportamiento emotivo racional. Manual del terapeuta. Barcelona: Océano.

Flett, G. \& Hewitt, P. (2004). The cognitive and treatment aspects of perfectionism: Introduction to the special issue. Journal of Rational-Emotive \& Cognitive-Behavior Therapy, 22(4), 229-236. https://doi.org/10.1023/B:JORE.0000047309.82574.fa 
Franco, K., Mancilla-Díaz, J., Álvarez, G. \& Vázquez, R. (2010) Estructura factorial y consistencia interna de la Escala Multidimensional de Perfeccionismo. Revista Mexicana de Psicología, 27(2), 143-149.

Freeman, J. (1998) Educating the Very Able: Current International Research. London: The Stationery Office.

Freeman, J. (2004). Giftedness and mild neurological disorders. ECHA News, 18, 6-8. Recuperado de http://www.joanfreeman.com/ pdf/G_and_disorder_ECHA_04.pdf

Frost, R., Marten, P., Lahart, C. \& Rosenblate, R. (1990). The dimensions of perfectionism. Cognitive Therapy and Research, 14(5), 449-46. https://doi.org/10.1007/BF01172967

Gagné, F. (2013). The DMGT: Changes Within, Beneath, and Beyond. Talent Development \& Excellence, 5(1), 5-19.

Geertz, C. (1987). La interpretación de las culturas. México: Gedisa.

González, M., Leal, D., Segovia, C. \& Arancibia, V. (2012). Autoconcepto y talento: una relación que favorece el logro académico.Psykche,21(1),37-53.https://doi.org/10.4067/s071822282012000100003

Hamachek, D. E. (1978). Psychodynamics of normal and neurotic perfectionism. Psychology, 15, 27-33.

Hewitt, P., Flett, G., Turnbull-Donovan, W. \& Mikail, S. (1991). The Multidimensional Perfectionism Scale: Reliability, Validity, and Psychometric Properties in Psychiatric Samples. A Journal of Consulting and Clinical Psychology, 3(3), 464-468. https://doi. org/10.1037/1040-3590.3.3.464

Kerr, B., Colangelo, N. \& Gaeth, J. (1988). Gifted Adolescents' Attitudes Toward Their Giftedness. Gifted Child Quarterly, 32(2), 245-247. Recuperadodehttp:/www.positivedisintegration.com/ Kerr1988.pdf. https://doi.org/10.1177/001698628803200201

Kim, J. \& Chang, E. (2010). The Conceptualization and Assessment of the Perceived Consequences of Perfectionism. (Tesis Doctoral) Universidad de Michigan, Estados Unidos. Recuperado de http:// deepblue.lib.umich.edu/bitstream/handle/2027.42/77633/ jeanmkim.pdf?sequence $=4$ 
Kreger, L. (1999). Perfectionism: The Crucible of Giftedness. Advanced Development, 8, 47-61. Recuperado de http://nmgifted.org/ GAC\%20Resources/Perfectionism\%20The\%20Crucible $\% 20$ of\%20Giftedness-SILVERMAN.pdf

Kornblum, M. \& Ainley, M. (2005). Perfectionism and the gifted: A study of an Australian school sample. International Education Journal, 6(2), 232-239.

Maia, B. R., Soares, M. J., Gomes, A., Marques, M., Pereira, A. T., Cabral, A. \& Macedo, A. (2009). Perfectionism in obsessive-compulsive and eating disorders. Revista Brasileira de Psiquiatría, 31(4), 322-327. https://doi.org/10.1590/S151644462009005000004

Parker, W. (1997). An Empirical Typology of Perfectionism in Academically Talented Children. American Educational Research Journal, 34(3), 545-562. https://doi.org/10.3102/00028312034003545

Robinson, N. M. (2008a). The social world of gifted children and youth. En S. I. Pfeiffer (Ed.), Handbook of giftedness in children: Psycho-educational theory, research, and best practices (pp. 33-52). New York, NY: Springer. https://doi.org/10.1007/978-0-38774401-8_3

Scappatura, M., Arana, F., Elizathe, L. \& Rutsztein, G. (2011). Perfeccionismo adaptativo y desadaptativo en trastornos alimentarios: un estudio de revisión. Anuario de investigaciones, 18, 81-88.

Schreier, M. (2014). Qualitative content analysis. En U. Flick (Ed.), The Sage Handbook of Qualitative Data Analysis (pp. 170-183). London: Sage. https://doi.org/10.4135/9781446282243.n12

Shafran, R., Cooper, Z. \& Fairburn, C. G. (2002). Clinical perfectionism: A cognitive-behavioural analysis. Behaviour research and therapy, 40(7), 773-791. https://doi.org/10.1016/S00057967(01)00059-6

Soenens, B., Elliot, A. J., Goossens, L., Vansteenkiste, M., Luyten, P. \& Duriez, B. (2005). The intergenerational transmission of perfectionism: parents' psychological control as an intervening variable. Journal of Family Psychology, 19(3), 358. https://doi. org/10.1037/0893-3200.19.3.358 
Speirs Neumeister, K. (2004). Factors Influencing the Development of Perfectionism in Gifted College Students. Gifted Child Quarterly, 48, 259-274. https://doi.org/10.1177/001698620404800402

Speirs Neumeister, K. \& Finch, H. (2006). Perfectionism in high-ability students: Relational precursors and influences on achievement motivation. Gifted Child Quarterly, 50(3), 238-251. https://doi. org/10.1177/001698620605000304

Stöeber, J. (2014). How Other-Oriented Perfectionism Differs from Self-Oriented and Socially Prescribed Perfectionism. Journal of Psychopathology and Behavioral Assessment, 36(2), 329-338. https://doi.org/10.1007/s10862-013-9397-7

Stöeber, J. \& Rambow, A. (2007). Perfectionism in adolescent school students: Relations with motivation, achievement, and wellbeing. Personality and Individual Differences, 42(7), 1379-1389. https://doi.org/10.1016/j.paid.2006.10.015

Stöeber, J. \& Childs, J. H. (2011). Perfectionism. In R. J. R. Levesque (Ed.), Encyclopedia of adolescence (Vol. 4, pp. 2053-2059). New York: Springer. https://doi.org/10.1007/978-1-4419-1695-2_279

Strauss, L. \& Corbin J. (1990). Basis of Qualitative Research. Londres: Sage.

Tarazona, J. (2011). Experiencia en la atención a alumnos con talento y superdotación en el ámbito escolar. CONSENSUS 16(1), 91-97.

Yin, R. (1989). Case Study Research: Design and methods. Londres: SAGE.

Recibido : 13 de junio, 2016

Revisado : 20 de enero, 2016 Aceptado : 24 de f ebrero, 2017 